\title{
Inflammatory Fibroid Polyp - a Rare Cause of Significant Gastric Bleeding
}

\author{
Tatjana Bogdanova*, Arnis Abolins*, Andrejs Vanags**, Ervins Vasko*, Janis Gardovskis**, Ilze Strumfa* \\ *Department of Pathology, Riga Stradins University, Latvia \\ ${ }^{* *}$ Department of Surgery, Riga Stradins University, Latvia
}

\section{Summary}

Bleeding, including gastrointestinal bleeding, is one of the most frequently encountered problems in surgical practice. The spectrum of causes comprises also an uncommon gastrointestinal pathology - inflammatory fibroid polyp. In order to diagnose the disease correctly and to choose the appropriate treatment, greater awareness of this rare entity is necessary.

Key words: inflammatory fibroid polyp, Vanek polyp, gastric bleeding.

\section{AIM OF THE DEMONSTRATION}

The aim of our report is to show a rare benign gastric lesion as the cause of gastrointestinal bleeding.

\section{CASE REPORT}

A 75-year-old lady was admitted to a university hospital because of gastric bleeding. Malignant tumour was suspected by the referring general practitioner. The patient complained about recurrent episodes of nausea and vomiting over the previous 6 months. She had also noticed dark discolouration of stools within the preceding three days. Physical investigation revealed pale, dry skin. The heart rate was regular, with the frequency 80 beats per minute. Arterial blood pressure was 130/85 mm Hg. Vesicular breath sounds with frequency 16 per minute were audible by lung auscultation. The abdominal palpation was painful in the epigastric region. Melaena was detectable per rectum. The test for occult blood in stools was positive. There was no evidence of hepatomegaly or splenomegaly. Laboratory findings showed anaemia. At admission, the red blood cell count was $3.80 \times 10^{12} / \mathrm{L}$ [laboratory reference

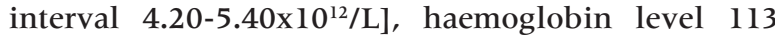
$\mathrm{g} / \mathrm{L}$ [120-160 g/L], white blood cell count 5.9×10 $/ \mathrm{L}$ $\left[4.0-10.0 \times 10^{9} / \mathrm{L}\right]$ and C-reactive protein level $4.0 \mathrm{mg} / \mathrm{L}$ [0-5.0 mg/L]. By upper gastrointestinal endoscopy, a mass measuring $3 \mathrm{~cm}$ was found in the posterior wall of gastric antrum (Fig.1A). The stalk was short and broad (Fig.1B); as it was difficult to visualise the stalk, no attempts of exact measurements were undertaken. The mass tended to slide into pylorus. Three biopsies were obtained from the lesion. The findings were suspicious of cancer but no strict proof of malignancy was obtained. There were no preoperative biopsy data about any non-epithelial proliferative process. By colonoscopy, diverticulosis of the sigmoid bowel was revealed. Biopsies were obtained from caecum and rectum and yielded no pathology.

The surgical treatment was done by upper middle laparotomy approach. Gastrotomy was performed by
$4 \mathrm{~cm}$ long incision in the anterior gastric wall. After that, polyp measuring $3 \mathrm{~cm}$ in diameter was directly seen in the posterior gastric wall. Thus, intraoperative endoscopy was not employed. Polyp excision with stitching at the base was done by TLC $75 \mathrm{~mm}$. The resection line was embedded then by continuous suture. The incision in the anterior gastric wall was closed by double suture, and additional ligatures were used for haemostasis. The removed gastric polyp was sent for histological examination. Grossly, it measured $3.0 \times 2.2 \times 2.0 \mathrm{~cm}$ and had short stalk measuring $1 \mathrm{~cm}$ in diameter and ending with the row of surgical staples. The distance between the pathological tissues and resection line was not evident grossly therefore multiple perpendicular sections were taken for microscopic evaluation and measurements. The lesion was characterized by proliferation of spindle cells showing perivascular onion-skin pattern and short fascicular architecture (Fig.2A). There was prominent infiltration of eosinophilic leukocytes between spindle cells (Fig.2B). The overlying mucosa was ulcerated (Fig.2C). The epithelium adjacent to ulceration showed reactive atypia and proliferation (Fig.2D). The spindle cell proliferation affected the mucosa, the markedly split lamina muscularis mucosae and submucosa. No capsule was present. Deeper gastric wall tissues as the muscular, subserosal and serosal layer were not present in the removed specimen. The resection line was free of pathological tissues; however, the distance between the advancing edge of the lesion and resection line varied between 0.1-0.6 $\mathrm{cm}$, as measured in appropriately marked histological specimens. By immunohistochemistry (IHC), the spindle cells showed diffuse cytoplasmic expression of vimentin and CD34 (Fig.3A-C), but absence of pan-cytokeratin, CD 117 (Fig.3D), CD31, DOG, actin, desmin, S-100 protein and HMB-45. The proliferation activity (by Ki-67, clone MIB-1) was $12 \%$. Based on histological and immunohistochemistry findings, inflammatory fibroid polyp s. Vanek polyp of gastric mucosa and submucosa was diagnosed. After the 
surgical treatment, patient was discharged following an uneventful post-operative recovery.

\section{DISCUSSION}

Inflammatory fibroid polyp (IFP) s. Vanek polyp represents rare gastrointestinal tract pathology. It was first described by Vanek in 1949 as a submucosal gastric granuloma with eosinophilic leukocyte infiltration. Six cases were reported then [9]. In 1953, Helwig and Ranier proposed the name "inflammatory fibroid polyp" [2]. IFP is a benign mesenchymal proliferative lesion, which originates from the submucosa [10]. Most (70-75\%) of the IFPs develop in the stomach, followed by small bowel $(23 \%)$. Occasional cases have been observed in the large bowel, gall bladder, oesophagus, duodenum and appendix [4]. Regarding the aetiology, triggers as a foreign body, parasite and Helicobacter pylori infection have been suggested [4]. Recently, neoplastic origin has been considered as activating mutations of platelet-derived growth factor receptor alpha gene have been reported in IFPs [3]. The average age of patients is 60 years, ranging 26-87 years [5]. The clinical manifestations usually are attributable to the pyloric obstruction, but occasionally diarrhoea or massive bleeding from polyp is observed $[1,6,8]$. IFPs tend to be small, measuring $1 \mathrm{~cm}$ in diameter. However, $4 \%$ of cases exceed $4 \mathrm{~cm}$ in diameter. IFPs develop from gastric submucosa as well-demarcated, oval or round, brown to gray, dense nodular masses with exophytic growth towards gastric lumen. Erosions of overlaying mucosa are seen in $26.8 \%$ cases, and ulcers develop in $3.5 \%$ of cases, mainly in larger lesions [1]. Clinically IFPs should be differentiated from other causes of gastrointestinal bleeding and gastric mass lesions. By IHC, the spindle cells of inflammatory fibroid polyp express vimentin, CD34 and cyclin D1, but lack CD 117 and DOG (in contrast to gastrointestinal stromal tumours. However, CD34 expression is shared between these entities), S-100 protein (in contrast to neural tumours) and cytokeratins contrasting to carcinoma [6]. IFPs are treated by local excision that can be performed by endoscopic or laparotomy access depending on the localization and size of polyp [7]. IFP is a benign process without metastatic potential. It does not recur after treatment. However, if the mass reaches significant size, it can lead to serious secondary complications.

In conclusion, inflammatory fibroid polyp should be included in the differential diagnosis of gastric bleeding and gastric mass lesions. Awareness of this rare pathology would be helpful in differential diagnosis to avoid over-diagnosing it as a malignant tumour. Limited surgical treatment is advisable in case of IFP in contrast to gastric cancer. IFP must be also differentiated from GIST by lack of CD 117 and DOG. Increased proliferation activity must not be interpreted as an evidence of malignancy in IFP.
Acknowledgements: This work was supported by the National Research Programme "Development of new prevention, treatment, diagnostics means and practices and biomedicine technologies for improvement of public health".

\section{REFERENCES}

1. Besson A, Saraga P. A case of a large inflammatory fibroid tumor of the gastric antrum (Vanek's tumor) // Schweiz Med Wochenschr, 1980; 110(25):990 993

2. Helwig EB, Ranier A. Inflammatory fibroid polyps of the stomach // Surg Gynecol Obstet, 1953; 96(3):335- 367

3. Huss S, Wardelmann E, Goltz D, Binot E, Hartmann W, Merkelbach-Bruse S, Buttner R, Schildhaus HU. Activating PDGFRA mutations in inflammatory fibroid polyps occur in exons 12, 14 and 18 and are associated with tumour localisation // Histopatology, 2012; 61(1):59-68

4. Jacobs TM, Lambrianides AL. Inflammatory fibroid polyp presenting as intussusception // Journal of Surgical Case Reports, 2013; 2:doi:10.1093/jscr/ rjt005

5. Liu TC, Lin MT, Montgomery EA, Singhi AD. Inflammatory fibroid polyps of the gastrointestinal tract: spectrum of clinical, morphologic, and immunohistochemistry features // Am J Surg Pathol, 2013; 37(4):586 - 592

6. Morales-Fuentes GA, de Arino-Suarez M, ZarateOsorno A, Rodriguez-Jerkov J, Terrazas-Espitia F, Perez-Manauta J. Vanek's polyp or inflammatory fibroid polyp. Case report and review of the literature // Cir Cir, 2011; 79(3):242 - 245,263 247

7. Paikos D, Moschos J, Tzilves D, Koulaouzidis A, Kouklakis G, Patakiouta F, Kontodimou K, Tarpagos A, Katsos I. Inflammatory fibroid polyp or Vanek's tumour // Dig Surg, 2007; 24(3): 231 - 233

8. Puri A, Gupta B, Bhalla S. Giant inflammatory fibroid polyp of stomach causing massive upper gastrointestinal bleeding // Indian J Gastroenterol, $1991 ; 10: 23-24$

9. Vanek J. Gastric submucosal granuloma with eosinophilic infiltration // Am J Pathol, 1949; 25(3):397 - 411

10. Wille P, Borchard F. Fibroid polyps of intestinal tract are inflammatory-reactive proliferations of CD34 positive perivascular cells // Histopathology, 1998, $32: 498-502$

\section{Address:}

Tatjana Bogdanova,

Department of Pathology, Riga Stradins University, Dzirciema Street 16, LV 1007, Riga, Latvia;

E-mail: Tatjana.Bogdanova@rsu.lv 


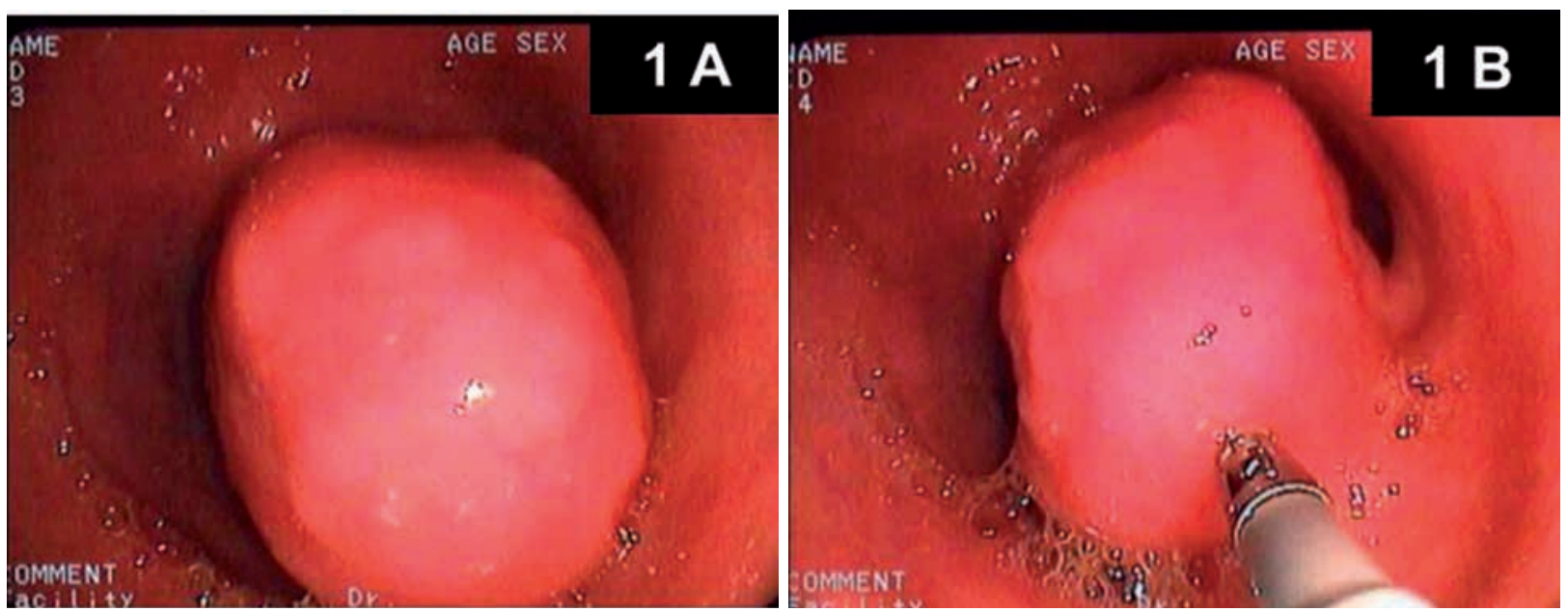

Fig. 1. Endoscopic view of the gastric mass lesion. A, Smooth, glistening red surface. B, Short and broad stalk
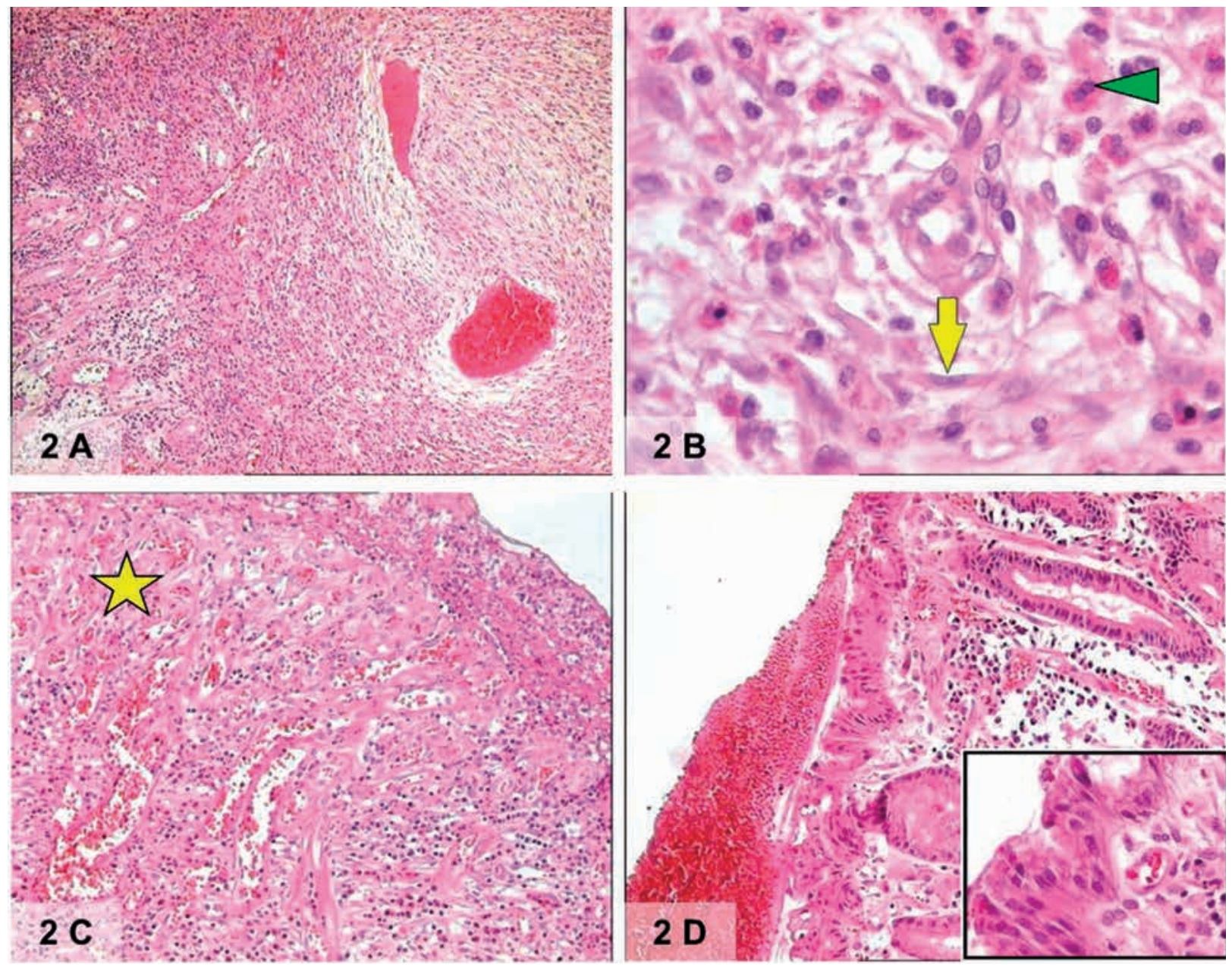

Fig. 2. Tissue structure of the inflammatory fibroid polyp. A, Perivascular onion-skin pattern. Note also the growth towards mucosa. B, Rich infiltration of eosinophilic leukocytes (arrowhead) between polyp-forming spindle cells (arrow). C, Ulcerated surface showing presence of granulation tissue (star). D, Reactive epithelial proliferation and atypia adjacent to the ulcer. Note the blood clot on the surface. Inset, Epithelial nuclear structure showing reactive changes only. Haematoxylin - eosin, original magnification: A, 50x; B, 400x; C, 50x; D, 100x; inset, 400x 

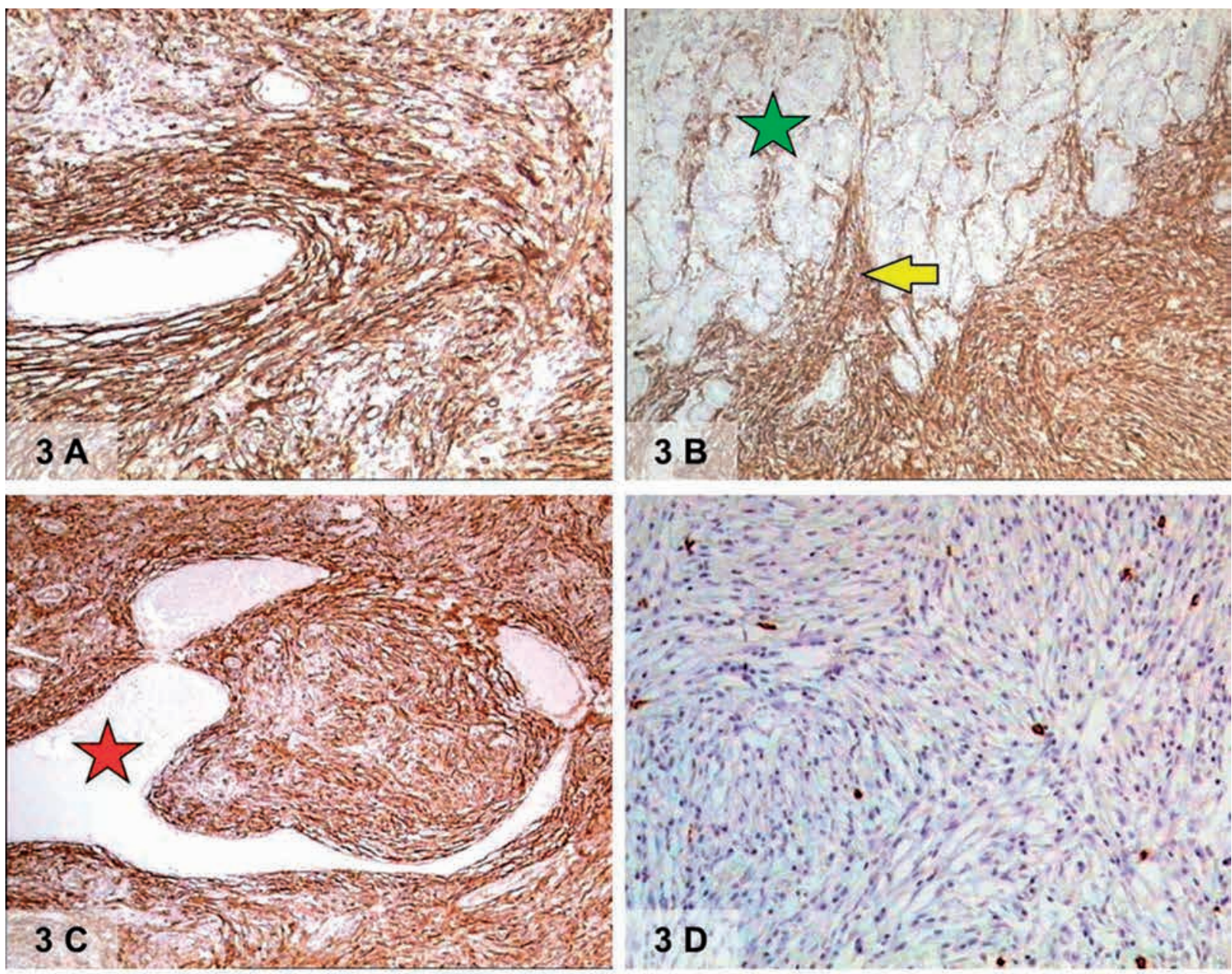

Fig. 3. Immunohistochemical features of inflammatory fibroid polyp. A, Intense cytoplasmic CD34 expression in the inflammatory fibroid polyp. Note the perivascular onion-skin pattern. B, Spread (arrow) into the mucosa (star). C, Deformation of large vessel (star). D, Lack of CD117 in the spindle cells of inflammatory fibroid polyp. Note the positive reaction in few infiltrating basophilic leukocytes. Immunoperoxidase, A-C, anti-CD34; D, anti-CD 117; original magnification: A-B, 100x; C, 50x; D, 400x 